Kennedy CR. The presenting features of brain tumours: a review of 200 cases. Arch Dis Child June 2006;91:502-506). (Respond: Dr CR Kennedy, Paediatric Neurology, University of Southampton, Mailpoint 21, Child Health, Southampton General Hospital, Southampton SO16 6YD, UK).

COMMENT. Several similar studies of presenting symptoms of brain tumors in children are reported in the pre-medline literature, notably the classic monograph by Chicago authors, Bailey P, Buchanan DN, and Bucy PC. Intracranial Tumors of Infancy and Childhood. Chicago and London, University of Chicago Press, 1939. In the present report, the earliest reference cited was 1986, and the majority was published in the late 1990s and after 2000. Many of the authors' findings corroborate those of earlier studies: 1) the incidence of seizures of $15 \%$ in children of all ages, with lesser frequency of $7 \%$ in children less than 3 years of age, and 2) delay in diagnosis of low grade vs high grade tumors and supratentorial vs infratentorial tumors. Relatively less recognized early manifestations of brain tumor emphasized in the present study include visual symptoms and learning and behavioral disorders, including lethargy.

In a study of 291 consecutive children treated for intracranial tumor at the Mayo Clinic from 1950-59, seizures occurred in $17 \%$ of the total group (in $25 \%$ of patients with supratentorial tumors and $12 \%$ of those with infratentorial tumors). (Backus RE, Millichap JG. Pediatrics 1962;29:978-984). The diagnosis of supratentorial tumors was delayed for an average of 2 years, whereas infratentorial tumors were diagnosed within 3 months of the initial seizure. Seizures were more common in patients with slowly growing grades 1 and 2 than rapidly expanding grades 3 and 4 astrocytomas (67\% vs $10 \%$ incidence). Diagnosis was facilitated by symptoms of increased intracranial pressure associated with the first seizure in $79 \%$ of infratentorial vs $20 \%$ of supratentorial tumors. In a report of presenting features of brain tumors in 21 infants (Gordon GS et al. Arch Dis Child 1995;73:345-347), vomiting in $9(43 \%)$ and large head in $16(76 \%)$ were the most common findings. Meningitis was suspected initially in $5(24 \%)$ infants with brain tumor, one with nuchal rigidity, and a CSF pleocytosis and elevated protein on premature spinal tap led to incorrect treatment with antibiotics and delay in CT scan and diagnosis.

\title{
POST-SURGICAL SPONTANEOUS REGRESSION OF RESIDUAL CEREBELLAR ASTOCYTOMA
}

Spontaneous regression of residual cerebellar astrocytoma following subtotal resection is reported in a 2-year-old boy followed for 11 years at the University of British Columbia, Canada. Tumor tissue left adjacent to the lateral wall of the fourth ventricle because of intraoperative bradycardia remained stable for 2 years. Between 2 and 7 years, repeated CT scans showed progressive decrease in size of the tumor, which became more cystic in appearance. The most recent scan at 11 years post-surgery showed complete regression of the tumor. Clinically, the child remained stable. The time course of regression is thought to rule out ischemic necrosis caused by surgery, and apoptosis is a more likely mechanism. (Steinbok P, Poskitt K, Hendson G. Spontaneous regression of cerebellar astrocytoma after subtotal resection. Childs Nerv Syst June 2006;22:572-576). (Respond: Dr P Steinbok, Division of Neurosurgery, Department of Surgery, University of British Columbia, Vancouver, BC, Canada). 